\title{
Reply to "The Neutrophil-to-Lymphocyte Ratio: An Ideal Marker for Early Diagnosis and Short-Term Prognosis of Acute Kidney Injury?"
}

\author{
Jiefu Zhu ${ }^{a}$ Zheng Dong ${ }^{a, b}$ \\ aDepartment of Nephrology, The Second Xiangya Hospital at Central South University, Changsha, China; \\ ${ }^{b}$ Department of Cellular Biology and Anatomy, Medical College of Georgia at Augusta University and Charlie \\ Norwood VA Medical Center, Augusta, GA, USA
}

We thank Drs. Lang and Schiffl for their interest in our recent study titled "Red Blood Cell Distribution Width and Neutrophil-to-Lymphocyte Ratio in Predicting Adverse Outcomes of Acute Kidney Injury in Hospitalized Patients" [1]. In this study, we provided evidence that neutrophil-to-lymphocyte ratio (NLR) and red blood cell distribution width (RDW) may have prognostic value for acute kidney injury (AKI) patients. Before going to details, we would make the statement that we essentially agree with Drs. Lang and Schiffl that NLR and RDW are not ideal or perfect biomarkers, rather they may be useful additions to the assessment and molecular parameters currently used in clinical settings.

In this retrospective, observational and cross-sectional study, it was difficult to control other potential confounding factors such as inflammation, despite conducting multivariate analysis. In addition, very few biomarkers of AKI were tested simultaneously with RDW or NLR in the patient cohort, making it impossible to compare with other biomarkers in this study. As such, our results do not negate the need and importance of clinical assessment in decision-making for the initiation of RRT. Currently, no single biomarker or test has an efficient predictive ability for patients at risk for AKI, progression of AKI, or mor-

karger@karger.com www.karger.com/kdd

Karger"

BOPEN ACCESS
(C) 2021 The Author(s)

Published by S. Karger AG, Basel

This is an Open Access article licensed under the Creative Commons Attribution-NonCommercial-4.0 International License (CC BY-NC) (http://www.karger.com/Services/OpenAccessLicense), applicable to the online version of the article only. Usage and distribution for commercial purposes requires written permission. tality from AKI. Our analyses suggest that increased NLR and/or RDW is an independent risk factor for AKI progression, providing additional information for risk stratification. In this aspect, NLR and RDW are readily available for most patients in their blood cell count reports. Therefore, NLR and RDW may be used along with clinical assessment and other biomarkers, as suggested by our study and those of others [1-4].

The value of NLR was calculated by dividing the counts of neutrophils by lymphocytes. Different labs use different methods for measuring neutrophils and lymphocytes, resulting in a variation in the thresholds for high NLR among different studies. In our study, the cutoff of NLR was determined by receiver-operating curves instead of referring to previous studies. We acknowledge that NLR cutoff values may vary in different studies or hospitals, so interpreting thresholds for high NLR should be based on the cohort measurement instead of a defined value from previous studies.

The cause of AKI is heterogeneous and includes sepsis, renal ischemia, and exposure to nephrotoxic drugs, to name a few. In this study, we tried to include as many as possible of demographic factors and clinical characteristics and made multivariate analysis to test the prognostic 
ability of NLR. The prognostic ability of NLR in AKI patients was valid, although demographic and clinical factors were needed to provide more accurate and specific information.

Selection bias may not be a significant factor in our study because we analyzed all hospitalized patients from January 1, 2016, to June 30, 2016, in the Second Xiangya Hospital and all AKI patients were identified according to the KDIGO guidelines in 2012 [5] except urine output criteria. The cutoff value of NLR was proposed in our study. The reference range of NLR in healthy population was not determined in this study because healthy subjects were not included.

Very few studies compared NLR with established biomarkers of inflammation or infection. In the future, a prospective study may investigate the relationship. Our study suggested the predictive ability of NLR in AKI patients. NLR has been reported as a predictor and prognostic marker in other diseases $[6,7]$, although the underlying mechanism remains unclear.

Finally, we would reiterate that we essentially agree with the comments by Drs. Lang and Schiffl. Our work indicates NLR is a risk factor for predicting RRT requirement, but this does not mean NLR can be used as a single, predictive biomarker. It is important to use it in combination with clinical assessment and related laboratory tests.

\section{Acknowledgements}

The authors sincerely thank the patients who participated in this study and the medical staff who took care of the patients at the Second Xiangya Hospital, Central South University.

\section{Statement of Ethics}

This research was approved by the Ethics Committee of the Second Xiangya Hospital at Central South University (Protocol Reference No. 2013-050-01). Written informed consent was not required because of the nonintervention and retrospective chart review design.

\section{Conflict of Interest Statement}

The authors have no conflicts of interest to declare.

\section{Funding Sources}

This work was supported partly by the grants from the National Key R\&D Program of China (2018YFC1312700) and the National Natural Science Foundation of China.

\section{Author Contributions}

J.Z. drafted the manuscript, and Z.D. revised the manuscript.

\section{References}

1 Zhu J, Zeng C, Zhang L, Shu S, Liu Y, Chen G, et al. Red blood cell distribution width and neutrophil-to-lymphocyte ratio in predicting adverse outcomes of acute kidney injury in hospitalized patients. Kidney Dis. 2020;6(5): 371-81.

2 Sicaja M, Pehar M, Derek L, Starcevic B, Vuletic V, Romic Z, et al. Red blood cell distribution width as a prognostic marker of mortality in patients on chronic dialysis: a single center, prospective longitudinal study. Croat Med J. 2013;54(1):25-32.
3 Kocyigit I, Eroglu E, Unal A, Sipahioglu MH, Tokgoz B, Oymak O, et al. Role of neutrophil/ lymphocyte ratio in prediction of disease progression in patients with stage- 4 chronic kidney disease. J Nephrol. 2013;26(2):358-65.

4 Chen D, Xiao D, Guo J, Chahan B, Wang Z. Neutrophil-lymphocyte count ratio as a diagnostic marker for acute kidney injury: a systematic review and meta-analysis. Clin Exp Nephrol. 2020;24(2):126-35.

5 Kidney Disease: Improving Global Outcomes (KDIGO) Group. KDIGO clinical practice guideline for acute kidney injury. Kidney Int Suppl. 2012.
6 Mei Z, Shi L, Wang B, Yang J, Xiao Z, Du P, et al. Prognostic role of pretreatment blood neutrophil-to-lymphocyte ratio in advanced cancer survivors: a systematic review and meta-analysis of 66 cohort studies. Cancer Treat Rev. 2017;58:1-13.

7 Pichler M, Hutterer GC, Stoeckigt C, Chromecki TF, Stojakovic T, Golbeck S, et al. Validation of the pre-treatment neutrophil-lymphocyte ratio as a prognostic factor in a large European cohort of renal cell carcinoma patients. Br J Cancer. 2013;108(4):901-7. 